\title{
Perceptions and Satisfaction of Saudi Medical Students with Neurology Education: A National Exploratory Study
}

\author{
Raseel Abdulaziz Aljthalin ${ }^{1, *}$, Raneem Abdulaziz Aljthalin ${ }^{1}$, Arwa Suliman Aljagwani ${ }^{1}$, Dareen Abdulelah Alanazi ${ }^{1}$, \\ Basel Abdullah Almeflh ${ }^{2}$, Abdulrahman Obaid Alharbi ${ }^{2}$ \\ ${ }^{1}$ College of Medicine, Majmaah University, Al Majmaah, Saudi Arabia \\ ${ }^{2}$ College of Medicine, King Saud University, Riyadh, Saudi Arabia
}

\section{ARTICLE INFO}

\section{Article History}

Received 08 June 2019

Accepted 14 August 2019

Keywords

Neurophobia

neurology education

Saudi medical students

\begin{abstract}
Most medical students perceive neurology as a difficult subject. Therefore, this results in students developing neurophobia. This study aimed to provide a national data source on the perceptions and satisfaction of Saudi medical students with neurology education. In 2018, an exploratory cross-sectional study was conducted among 3rd to 6th year Saudi medical students $(n=409)$. The study encompassed 29 medical schools in the Kingdom of Saudi Arabia. The students were recruited using cluster sampling of all regions of the Kingdom. The data were analyzed using descriptive statistics and analysis of variance. Based on a five-point Likert scale, the students had modest perceptions (mean $=2.91 \pm 0.78)$ and modest satisfaction levels $($ mean $=2.97 \pm 1.02)$ with neurology education. There were no significant differences in perceptions or satisfaction by sex, nationality, or university type. Nevertheless, highly significant differences in both perceptions and satisfaction across universities were found $(p=0.000)$. In general, Saudi medical students suffer from neurophobia. Given the implication of neurology and brain health, a national strategy is needed to address medical students' perceptions, knowledge, and skills with regard to neurology education. This is essential to promote career development needs among medical graduates, and to meet service demands for neurologists in Saudi Arabia. Furthermore, efforts are needed to align and reduce existing variations across medical schools in the Kingdom.
\end{abstract}

(c) 2019 Dr. Sulaiman Al Habib Medical Group. Publishing services by Atlantis Press International B.V. This is an open access article distributed under the CC BY-NC 4.0 license (http://creativecommons.org/licenses/by-nc/4.0/).

\section{INTRODUCTION}

Neurology is the branch of medicine that deals with disorders of the nervous system, including the brain, spinal cord, peripheral nerves, and muscles. There is a wide spectrum of neurological disorders, including manageable ones (e.g., migraine and epilepsy), acute emergencies (e.g., stroke and meningitis), and chronic disabling conditions (e.g., Parkinson's disease and multiple sclerosis) [1].

Throughout the world, the field of neurology is considered as one of the most difficult and least comprehended medical specialties [2]. It is believed that students perceive neural sciences and clinical neurology as complex, which contributes to the development of a sense of fear of these topics. This results in a syndrome known as neurophobia [2-11].

Perceiving difficulty in learning neurology is not a recent issue. More than two decades ago, a British survey among medical students estimated that approximately half of medical students experience neurophobia at a given time during their education [3]. Furthermore, the study identified three main issues related to learning neurology: the necessity to have good knowledge of basic

"Corresponding author. Email: raseel.1995@hotmail.com

Peer review under responsibility of the Dr. Sulaiman Al Habib Medical

Services Group Company neurosciences, the quality of teaching, and the difficulty of carrying out neurological examinations [3]. Surprisingly, subsequent and recent studies all over the world (e.g., American [4], Canadian [5], Chinese [6], Indian [7], Irish [8], British [9,10], and Saudi studies $[2,11])$ had comparable findings concerning difficulties with neurology education.

Regardless of future specialty choice, all medical student graduates should acquire basic neurology knowledge and skills that enable them to recognize and manage neurological problems of their patients. Neurologists are regularly needed to meet service demands in the Kingdom of Saudi Arabia (KSA). Therefore, it is important to explore the beliefs of Saudi medical students about neurology education at a national level in the KSA and to provide recommendations for improvement, if needed.

\subsection{Aims and Objectives}

This study aimed to provide a national data source on the perceptions and satisfaction of Saudi medical students with neurology education. The specific objectives were to determine students' perceptions and satisfaction with neurology education and to test differences in perceptions and satisfaction toward neurology education according to student's sex, nationality, academic level, university type, and specific university. 


\section{METHODOLOGY}

\subsection{Procedure}

In 2018, an exploratory cross-sectional study was conducted among 3 rd to 6 th year Saudi medical students. The study included 29 medical schools in the KSA. Among these schools, 24 (83\%) were public and five $(17 \%)$ were private. The students were recruited using cluster sampling of all regions in the KSA. Ethical approval to conduct the study was obtained from the University Ethical Committee of the Majmmah University.

The sample size was calculated as follows:

$$
N=\frac{z^{2} \times p q}{d^{2}} \rightarrow N=\frac{1.96^{2} \times(0.5)(0.5)}{0.05^{2}}=384
$$

A total of 409 students were enrolled to prevent high non-response rate and/or missing data.

The questionnaire was first provided to three academic experts to review the questions for content validity, clearness, and appropriateness. Then, the questionnaire was piloted on 25 students, who were selected from outside the study sample. The participants were informed that participation in the study was anonymous and voluntary.

\subsection{Questionnaire}

The study's 18-item questionnaire was designed and distributed in the form of a web link. Responses were collected over a period of 9 weeks. The questionnaire comprised the following parts.

\subsubsection{Sociodemographic and academic characteristics}

- Sex (male/female)

- Age (continuous variable)

- Nationality (Saudi/non-Saudi)

- Academic year (3rd to 6th year)

- Type of university (public/private)

\subsubsection{Students' perceptions about neurology education}

Students' perceptions were assessed using the following six statements: "I have learned to record a complete neurological history of a patient and to perform a complete neurological examination." "We have a qualified staff teaching neurology." "At the inpatient services at my university, I have been exposed to common neurological diagnoses and to more complex/rare conditions." "At the outpatient services at my university, I have been exposed to a broad range of disorders." "If I am offered an opportunity to undergo neurology training at a reputable neurology center, I will make neurology my career of choice." "I have intentions to pursue neurology as a future specialty option" (Cronbach's alpha $=0.71$ ). The responses were rated using a five-point Likert scale (strongly disagree to strongly agree).

\subsubsection{Students' satisfaction level with neurology education}

Students' satisfaction level was assessed using the following three question. "Overall, how satisfied are you with the module of neurology?" "Overall, how satisfied are you with the total contact hours you received in the module of neurology?" "Overall, how satisfied are you with the topics that you have learned in the module of neurology?" (Cronbach's alpha $=0.71)$. The responses were rated using a fivepoint Likert scale (ranging from strongly disagree to strongly agree).

\subsection{Statistical Analyses}

The data were entered and analyzed using SPSS version 23.0 (IBM Corp., Armonk, NY, USA). Frequencies and descriptive statistics were used to provide an overall picture of the sample population. Internal consistencies (reliability) of the overall perceptions and satisfaction with neurology education scales were calculated using Cronbach's alpha $(\alpha)$. Significant differences between study variables were assessed using analysis of variance. A $p$-value $<0.05$ was considered statistically significant.

\section{RESULTS}

\subsection{Characteristics of the Sample}

The study involved a total of 409 medical students with a response rate of $100 \%$. Approximately two-thirds of the students $(64.1 \%)$ were female. The average age was 22.6 years [standard deviation $(\mathrm{SD})=1.6]$. Most students $(95.8 \%)$ were Saudi nationals. Approximately one-third of the students (32.3\%) were in the 6 th academic year, and most of them (87\%) studied at public universities. Detailed characteristics of the sample are shown in Table 1.

\subsection{Students' Perceptions about Neurology Education}

The mean score of students' perceptions was $2.91(\mathrm{SD}=0.78)$.

Table 1 Demographic and academic characteristics of the sample population $(n=409)$

\begin{tabular}{lc}
\hline Character & $\begin{array}{c}\text { Total sample }(\boldsymbol{n}=\mathbf{4 0 9}) \\
\boldsymbol{n}(\%)\end{array}$ \\
\hline Sex & $147(35.9)$ \\
Male & $262(64.1)$ \\
Female & \\
Nationality & $392(95.8)$ \\
Saudi & $17(4.2)$ \\
Non-Saudi & \\
Academic year & $81(19.8)$ \\
3rd & $95(23.2)$ \\
4th & $101(24.7)$ \\
5th & $132(32.3)$ \\
6th & \\
University & $24(83)$ \\
Public & $5(17)$ \\
Private & $22.6 \pm 1.6$ \\
Age, mean \pm standard deviation &
\end{tabular}




\subsection{Students' Satisfaction with Neurology Education}

The mean score of students' satisfaction was $2.97(\mathrm{SD}=1.02)$.

\subsection{Differences in Perception of Neurology Education According to Study Variables}

As shown in Table 2, there were no significant differences in perception of neurology education by sex, nationality, academic year, or university type (all $p>0.05$ ).

\subsection{Differences in Satisfaction with Neurology Education According to Study Variables}

As shown in Table 3, there were no significant differences in satisfaction with neurology education by sex, nationality, or university type (all $p>0.05)$. There was a significant difference $(p=0.024)$ by academic year. Specifically, 4 th year students were the most satisfied, whereas 6th year students were the least satisfied.

\subsection{Differences in Perception of Neurology Education by Individual University}

There was a highly significant difference $(p=0.000)$ in perception of neurology education between individual universities. Among the 29 universities, students at Bish University, Narran University, and Albaha University had the highest positive perceptions, whereas students at Tiba University, Alfarabi Colleges, and Jeddah University had the lowest positive perceptions (see Figure 1).

\subsection{Differences in Satisfaction with Neurology Education by Individual University}

There was a highly significant difference $(p=0)$ in satisfaction with neurology education between individual universities. Among

Table 2 Differences in perception of neurology education by study variables

\begin{tabular}{lll}
\hline Demographic variable & Mean (SD) & $\boldsymbol{p}$ \\
\hline Sex & & \\
$\quad$ Male & $2.91(0.81)$ & 0.97 \\
$\quad$ Female & $2.91(0.73)$ & \\
Nationality & & \\
$\quad$ Saudi & $2.90(0.78)$ & 0.32 \\
$\quad$ Non-Saudi & $3.10(0.73)$ & \\
Academic year & & \\
$\quad$ 3rd & $2.88(0.75)$ & \\
4th & $3.08(0.70)$ & 0.93 \\
5th & $2.81(0.70)$ & \\
6th & $2.89(0.83)$ & \\
University & \\
$\quad$ Public & $2.93(0.77)$ & \\
$\quad$ Private & $2.80(0.86)$ & 0.217 \\
\hline
\end{tabular}

$\mathrm{SD}$, standard deviation
Table 3 Differences in satisfaction with neurology education by study variables

\begin{tabular}{llc}
\hline Demographic variable & Mean (SD) & $\boldsymbol{p}$ \\
\hline Sex & & \\
$\quad$ Male & $3.06(1.09)$ & 0.149 \\
$\quad$ Female & $2.92(1.21)$ & \\
Nationality & & \\
$\quad$ Saudi & $2.96(1.02)$ & 0.20 \\
$\quad$ Non-Saudi & $3.16(1.02)$ & \\
Academic year & & \\
$\quad$ 3rd & $2.92(0.98)$ & \\
$\quad$ 4th & $3.22(0.98)$ & $\mathbf{0 . 0 2 4}$ \\
5th & $3.00(0.98)$ & \\
$\quad$ 6th & $2.81(1.1)$ & \\
University & \\
$\quad$ Public & $2.97(1.02)$ & 0.839 \\
$\quad$ Private & $2.94(1.03)$ & \\
Significant differences are shown in bold $(p<0)$. SD, standard deviation.
\end{tabular}

the 29 universities, students at Prince Sattam Bin Abdulaziz University, Imam Muhammad Ibn Saud University, and Najran University had the highest satisfaction, whereas students at Tiba University, Alfarabi colleges, and Tabouk University had the lowest satisfaction (see Figure 2).

\section{DISCUSSION}

Our study aimed to explore the beliefs of Saudi medical students about neurology education.

The first objective of the study was to determine students' perceptions and satisfaction with neurology education. The results of this study indicated that Saudi medical students held moderately positive perceptions as well as moderate levels of satisfaction with neurology education. Modest levels of positive perceptions and satisfaction with neurology education among medical students were reported and are consistent with previous national and international studies. For example, a study among American medical students [6] reported that students encountered difficulty with neurology and demonstrated less self-confidence in dealing with neurological complaints. Likewise, Chinese students demonstrated a low knowledge of neurology, and ranked the neurology field as the last to specialize in the future [8]. Canadian students also shared comparable fears of neurology, where approximately half of the students (46\%) considered clinical neurology as one of the most difficult disciplines in medicine [7]. The reasons why Canadian students developed neurophobia were explained by the relatively short time frame during which the content is delivered to students and by the failure to apply clinical training when basic neuroscience concepts are taught [7]. In addition, approximately half $(43 \%)$ of Indian students were found to have neurophobia and reported that the major reason why they would not take neurology in the future is the degenerative nature of neurological diseases [8].

Previous Saudi studies also showed comparable findings. A study among the students at King Abdulaziz University in Jeddah reported that most students $(84 \%)$ perceived neurology as a difficult topic, and approximately one-third of the students (30\%) were not satisfied with their neurology teaching experience [2]. In a study among the students at University of Tabuk [8], approximately 


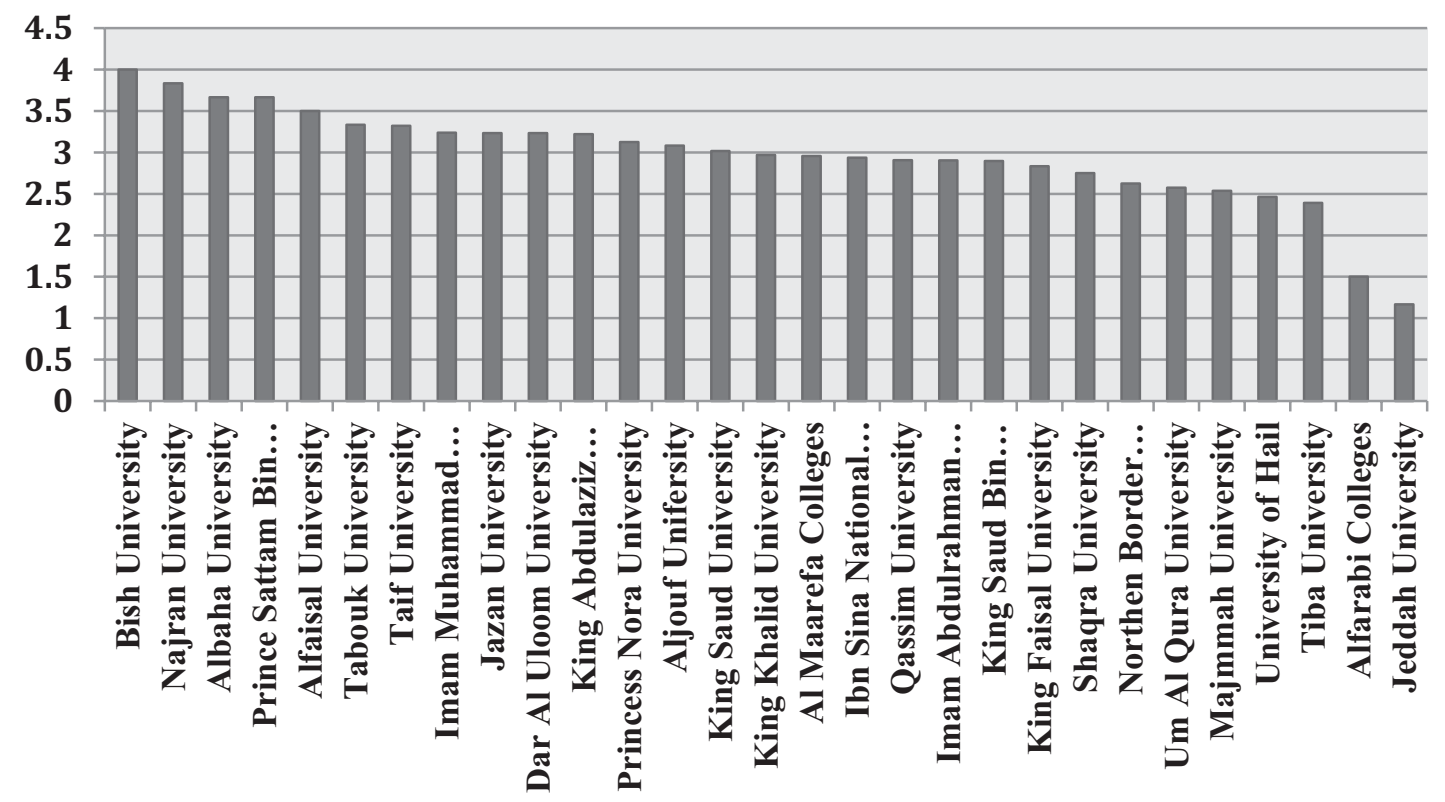

Figure 1 Differences in perceptions with neurology education by individual university.

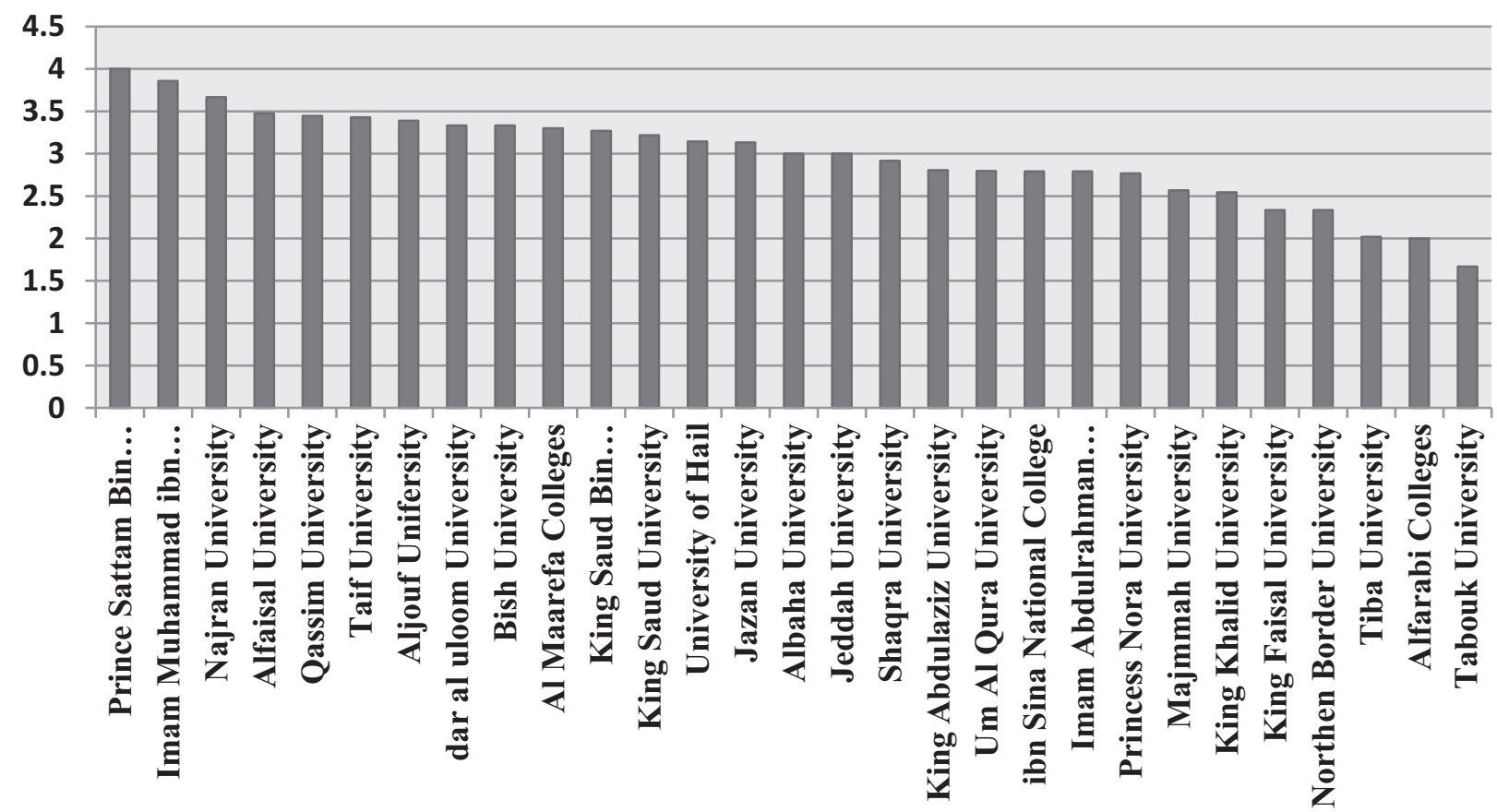

Figure $2 \mid$ Differences in satisfaction with neurology education by individual university.

one-third of the students (31\%) reported that neurosurgery was the most difficult subject for them. Furthermore, the most frequently reported factors for perceiving these difficulties were the incompetent teaching and the difficult nature of neuroanatomy.

Given the importance of neurology and brain health, a national strategy is needed to address medical students' perceptions, knowledge, and skills with regard to neurology education to promote career development needs. Furthermore, the responsibility of teaching staff members as positive role models in neurology education should be highlighted.
The second objective of the study was to test differences in perceptions and satisfaction with neurology education according to student's sex, nationality, academic level, university type, and individual university. The results showed no significant differences in perceptions or satisfaction by sex, nationality, or university type. Nevertheless, 4th year students were the most satisfied, whereas 6th year students were the least satisfied. Moreover, significant differences in both perceptions and satisfaction across universities were found. These differences may be attributable to variations in the teaching staff and teaching strategies. Therefore, efforts are needed to align and reduce existing variations across medical schools in the KSA. 
Our results have revealed high prevalence of neurophobia among Saudi medical students. Given the implication of neurology and brain health, a national strategy is needed to address medical students' perceptions, knowledge, and skills with regard to neurology education. This is essential to promote career development needs among medical graduates, and to meet service demands for neurologists in the whole kingdom. Furthermore, efforts are needed to align and reduce existing variations across medical schools in the KSA.

The major limitation of this study is that we only assessed students' perceptions and satisfaction with neurology without considering their academic performance. Further research is required to determine whether negative perceptions and low satisfaction are coupled with poor academic and clinical performance. Nonetheless, this study is important because it provides a national and up-to-date picture of the status of neurology education in the KSA as a whole.

\section{CONFLICTS OF INTEREST}

The authors declare they have no conflict of interest.

\section{AUTHORS' CONTRIBUTION}

RAA participated in data collection, literature review, data analysis, and wrote parts of Introduction and Discussion sections. RAA and ASA were involved in data collection, literature review, and wrote parts of Introduction and Discussion sections. DAA and BAA were involved in data collection, data entry, and analysis in the Results section. AOA. performed step-by-step follow-up and reviewed and revised the whole paper.

\section{REFERENCES}

[1] Pickersgill T. A career in neurology. BMJ 2004;329;s93.

[2] Abulaban AA, Obeid TH, Algahtani HA, Kojan SM, Al-Khathaami AM, Abulaban AA, et al. Neurophobia among medical students. Neurosciences (Riyadh) 2015;20;37-40.

[3] Jozefowicz RF. Neurophobia: the fear of neurology among medical students. Arch Neurol 1994;51;328-9.

[4] Zinchuk AV, Flanagan EP, Tubridy NJ, Miller WA, McCullough LD. Attitudes of US medical trainees towards neurology education: "Neurophobia" - a global issue. BMC Med Educ 2010;10;49.

[5] Fantaneanu TA, Moreau K, Eady K, Clarkin C, DeMeulemeester C, Maclean H, et al. Neurophobia inception: a study of trainees' perceptions of neurology education. Can J Neurol Sci 2014;41;421-9.

[6] Lukas RV, Cooper B, Morgan I, Brorson JR, Dong H, Sherer R. Attitudes toward neurosciences in medical students in Wuhan, China: a survey study. World Neurosurg 2014;82;266-9.

[7] Gupta NB, Khadilkar SV, Bangar SS, Patil TR, Chaudhari CR. Neurology as career option among postgraduate medical students. Ann Indian Acad Neurol 2013;16;478-82.

[8] Flanagan E, Walsh C, Tubridy N. 'Neurophobia' - attitudes of medical students and doctors in Ireland to neurological teaching. Eur J Neurol 2007;14;1109-12.

[9] Pakpoor J, Handel AE, Disanto G, Davenport RJ, Giovannoni G, Ramagopalan SV. National survey of UK medical students on the perception of neurology. BMC Med Educ 2014;14;225.

[10] Schon F, Hart P, Fernandez C. Is clinical neurology really so difficult? J Neurol Neurosurg Psychiatry 2002;72;557-9.

[11] Alhejaili MA, Alrashedi MH, Alatawi AN, Alenezi MF, Albalawi KA, Albalawi MF. Assessment of attitude and perception toward neurology and neurosurgery specialties among medical students and interns attending College of Medicine at University of Tabuk in Tabuk City, Saudi Arabia-2017. Egypt J Hosp Med 2018;71;2960-2. 